\title{
TEMPERATURE, ACCEPTOR CONCENTRATION AND DONOR CONCENTRATION DEPENDENCY OF ELECTRONIC MOBILITY IN BULK GAN AND GAP
}

\author{
Md. Rabiul Islam ${ }^{1 *}$ and Md. Zahid Hasan Mahmood ${ }^{2}$ \\ ${ }^{1,2}$ Department of Applied Physics Electronics and Communication Engineering, \\ University of Dhaka, Bangladesh
}

\begin{abstract}
Mobility is an important factor to determine the speed of an electronic device. Devices composed of electronic materials with higher mobility are able to achieve higher speed. It is therefore favourable to maximize the mobility. In this paper, we have chosen GaN and GaP as semiconductor material and Lowfield electron mobility has been calculated in bulk and wurzite phase GaN and GaP. The dependency of mobility upon temperature is shown. Beside this, dependency of mobility upon acceptor concentration and donor concentration are also shown.
\end{abstract}

\section{KEYWORDS}

Mobility, Scattering, GaN, GaP, Donor, Acceptor

\section{INTRODUCTION}

The tremendous theoretical enrichment and technological development in the field of semiconductor has made it possible of wide scale application in wireless communication, high power devices, optoelectronic and high speed devices. High-speed devices like gunn diode, microwave transistor, impact-ionization avalanche transit-time (IMPATT) diode, resonanttunneling diode (RTD) are ultimate result of this rapid development in semiconductor research field. The key requirement of these high speed devices is high electron mobility. Therefore to achieve better and better performance from high-speed devices continuation of research to achieve further high mobility is required $[1,2]$.

However, till the last decade primary semiconductor were Silicon ( $\mathrm{Si}$ ) and Group III-V materials such as Gallium Arsenide (GaAs) and Aluminium Arsenide (AlAs). Although these materials had extensive application in various fields, those materials have a major limitation of narrow band gaps (1.1 eV for $\mathrm{Si}$ and $1.4 \mathrm{eV}$ for GaAs). These limitations have made their usage limited. It is because of the fact that electrons can easily travel from the valence band to the conduction band in a material with a narrow band gap. Those materials are therefore not suitable for high temperature and high speed applications [3]. The $\mathrm{GaN}$ and $\mathrm{GaP}$ have band gap of $3.4 \mathrm{eV}$ and 2.25 $\mathrm{eV}$ respectively at $300 \mathrm{~K}$. This wide band gap and other properties have made those materials specially applicable for exciting applications [4]. 


\section{MoBILITY}

Mobility is a measure of how well charge carriers are able to move through a substance. Electrons are able to flow more quickly in materials with higher mobility [5]. Bulk mobility measures how well charge carriers move through bulk semiconductors and it is important to study electrical properties of semiconductor. The mobility is determined by the rate at which electrons are scattered by impurities and defects within the crystal structure of the semiconductor. This rate is the reciprocal of the relaxation time $\tau$, the average time between collisions. The relaxation time can be adjusted to account for the varying degrees of scattering on any given collision. This is called the momentum relaxation time $\tau_{\mathrm{m}}$. The mobility is dependent upon $\tau_{\mathrm{m}}$ by the equation [6],

Where,

$$
\mu=\frac{\mathrm{e} \tau_{\mathrm{m}}}{\mathrm{m}^{*}}
$$

- $\mathrm{e}$ is the charge of the electron.

- $\mathrm{m}^{*}$ is the effective mass of the electron.

\section{SCATTERINGS}

As free charge carriers traverse through a semiconductor, they encounter various scatterings [7]. Scatterings influence the bulk electronic mobility. The important mechanisms of electron scattering considered are:

\subsection{Ionized Impurity Scattering}

Carriers, electrons or holes, are scattered from impurities and defects. Some of these scatterers are charged and some are neutral. Defects, if charged, can be considered as ionized impurities for mobility calculation purposes. The ionized impurity scattering is dominant at low temperatures because, as the thermal velocity of the carriers goes down, the effect of long-range Coulombic interactions on their motion is increased [7,9]. Now the momentum relaxation time for ionized impurity scattering is given as [8],

Here,

$$
\tau_{i i}=\frac{0.414 \epsilon_{\mathrm{r}}^{2}(0) \mathrm{T}^{3 / 2}}{\mathrm{Z}^{2} \mathrm{~N}_{\mathrm{i}}\left[\ln (1+\mathrm{b})-\frac{\mathrm{b}}{1+\mathrm{b}}\right]}
$$

Where,

$$
\mathrm{b}=4.31 \times 10^{13}\left[\frac{\epsilon_{\mathrm{r}}{ }^{2}(0) \mathrm{T}^{2}}{\mathrm{n}^{*}}\right]\left(\frac{\mathrm{m}^{*}}{\mathrm{~m}}\right) \mathrm{x}
$$

- $\epsilon_{\mathrm{r}}(0)$ is static dielectric constant.

- $\mathrm{N}_{\mathrm{i}}$ is the concentration of ionized impurities.

- $\mathrm{m}$ is the mass of electron.

- $\mathrm{T}$ is the temperature in Kelvin.

\subsection{Neutral Impurity Scattering}

If the scatterers are neutral, they can be handled using the neutral impurity scattering theory. When an electron approaches close to a neutral atom it exchanges momentum with the bound electron. Note that, the scattering cross section for this process is not nearly as large as in the case of the ionized impurity scattering [7]. Considering neutral impurities scattering momentum relaxation time equation is written as [8], 
Where

$$
\tau_{\text {in }}=\frac{8.16 \times 10^{6}}{\epsilon_{\mathrm{r}}(0) \mathrm{N}_{\mathrm{n}}}\left(\frac{\mathrm{m}^{*}}{\mathrm{~m}}\right)^{2}
$$

- $\mathrm{N}_{\mathrm{n}}$ represents the concentration of neutral impurities.

\subsection{Acoustic Deformation Potential Scattering}

Beginning at about $5 \mathrm{~K}$, acoustic phonon scattering becomes the main mechanism limiting the mobility through both deformation potential and piezoelectric scattering. Unless explicitly specified, acoustic phonon scattering is generally assumed to be only because of the deformation potential as the nomenclature was developed for semiconductors with no or negligible piezoelectric behavior. Because the nitrides are highly piezoelectric, both components must be taken into consideration $[8,9]$.

For temperatures below about $200 \mathrm{~K}$, the deformation potential induced acoustic scattering dominates over the piezoelectric components. For high temperatures, the piezoelectric component is relatively stronger [7]. Considering acoustic deformation potential scattering momentum relaxation time equation is written as [8],

Where,

$$
\begin{gathered}
\tau_{\mathrm{dp}}=\frac{2.4 \times 10^{-20} \mathrm{C}_{\mathrm{L}}}{\mathrm{D}_{\mathrm{ap}}{ }^{2} \mathrm{~T}^{3 / 2}}\left(\frac{\mathrm{m}}{\mathrm{m}^{*}}\right)^{1 / 2} \\
\mathrm{C}_{\mathrm{L}}=\frac{1}{5}\left(3 \mathrm{C}_{11}+2 \mathrm{C}_{12}+4 \mathrm{C}_{44}\right)
\end{gathered}
$$

- $\mathrm{D}_{\mathrm{ap}}$ is the deformation potential.

- $\mathrm{C}_{\mathrm{L}}$ elastic constant (Logarithmic component).

\subsection{Piezoelectric Scattering}

In non-centrosymmetric crystals, a polarization field is induced when stress is applied. Carriers then interact with the electric field induced by strain. In a sense, the carriers can be scattered by a TA phonon in addition to LA phonons through the piezoelectric coupling, as is the case in the deformation potential acoustic phonon scattering [7, 9]. For piezoelectric scattering the momentum relaxation time equation is given as [8]

Here,

$$
\tau_{\text {ip }}=\frac{9.54 \times 10^{-8}}{\mathrm{~h}_{14}{ }^{2}\left(\frac{3}{c_{\mathrm{L}}}+\frac{3}{c_{\mathrm{T}}}\right) \mathrm{T}^{3 / 2}}\left(\frac{\mathrm{m}}{\mathrm{m}^{*}}\right)^{1 / 2}
$$

$$
\begin{aligned}
& \mathrm{C}_{\mathrm{L}}=\frac{1}{5}\left(3 \mathrm{C}_{11}+2 \mathrm{C}_{12}+4 \mathrm{C}_{44}\right) \\
& \mathrm{C}_{\mathrm{T}}=\frac{1}{5}\left(3 \mathrm{C}_{11}-\mathrm{C}_{12}+3 \mathrm{C}_{44}\right)
\end{aligned}
$$

Where,

- $\mathrm{h}_{14}$ is the piezoelectric stress tensor.

- $\mathrm{c}_{\mathrm{T}}$ is the elastic constant (Transverse component).

\subsection{Optical Deformation Potential Scattering}

Optical phonons in semiconductors have energies in the tens of mill-electron volts with the figure for LO phonons to be about $92 \mathrm{meV}$ [7]. This implies that at low temperatures, such as $100 \mathrm{~K}$, most electrons do not have sufficient thermal energy to emit optical phonons. Moreover, the thermal occupation number for phonons is very small and therefore the probability of an electron 
absorbing an optical phonon is very low. This basically indicates that optical phonon scattering at low temperatures is negligible $[10,11]$.

At higher temperature the picture is very different in the sense that electrons have sufficient energy to emit optical phonons and overtake LA acoustic phonon scattering. This statement is particularly applicable to polar semiconductors where the Frohlich electron-phonon coupling is very strong scattering by LA phonons relaxes primarily the electron momentum with negligible energy change; however, scattering by optical phonons relaxes both electron momentum and energy [7]. The momentum relaxation time for optical phonon scattering by deformation potential is given by [8],

$$
\tau_{\mathrm{op}}=\frac{4.83 \times 10^{-20} \mathrm{C}_{\mathrm{L}}\left[\mathrm{e}^{\frac{\theta}{\mathrm{T}}}-1\right]}{\xi_{\mathrm{A}}{ }^{2} \mathrm{~T}^{1 / 2} \theta}\left(\frac{\mathrm{m}}{\mathrm{m}^{*}}\right)^{3 / 2}
$$

Here,

$$
\theta=\frac{\mathrm{h} \omega_{\mathrm{LO}}}{\mathrm{k}}
$$

Where,

- $\xi_{\mathrm{A}}$ denotes the optical phonon deformation potential.

- $\mathrm{h} \omega_{\mathrm{LO}}$ is the optical phonon energy.

- $\omega_{\mathrm{LO}}$ is the optical phonon frequency.

- $\mathrm{k}$ is the Boltzmann constant.

- $h$ is the Plank constant.

\section{RESULTS}

The results obtained are shown in the following subsections. A comparison between results obtained in the case of $\mathrm{GaN}$ and $\mathrm{GaP}$ is also done.

\subsection{Temperature Dependency of Electronic Mobility}

Figure 1 depicts the variation of low field electronic mobility as a function of temperature for $\mathrm{GaN}$ and $\mathrm{GaP}$, which also includes the contribution of individual scattering mechanism in mobility.

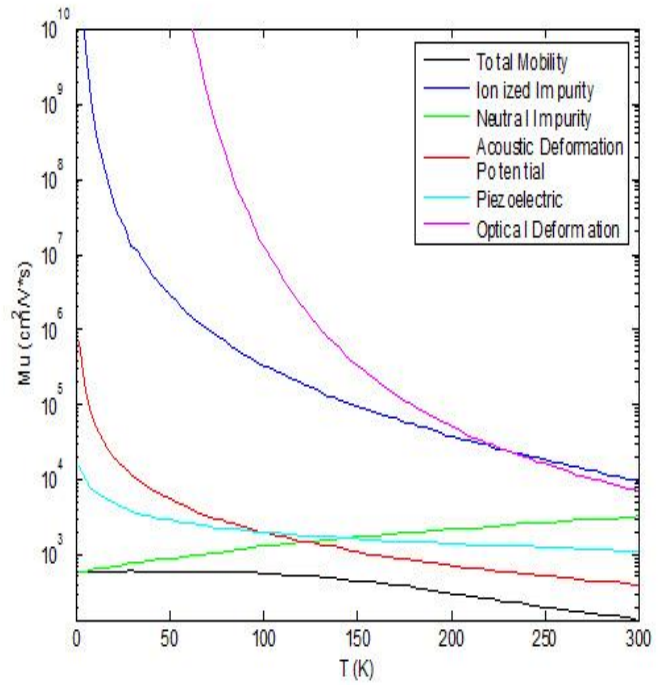

(a)

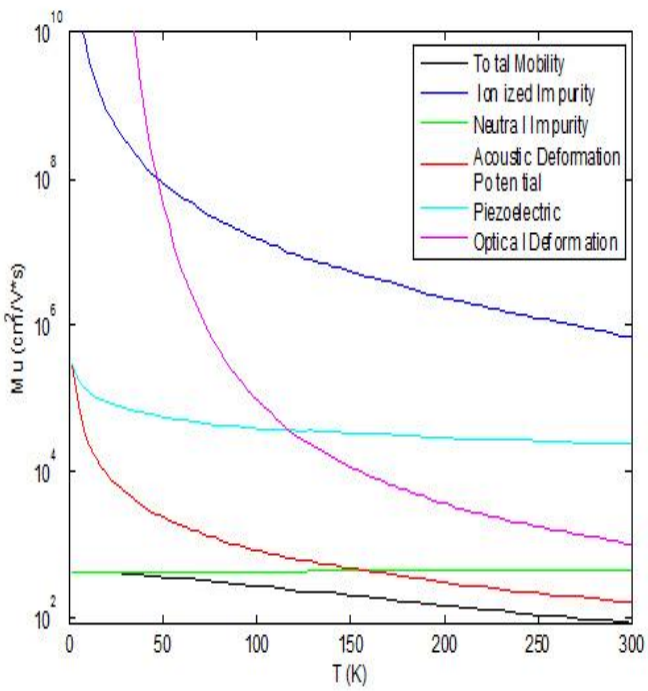

(b)

Figure 1. Temperature dependency of mobility, (a) for $\mathrm{GaN}$ and (b) for GaP. 


\subsection{Acceptor Concentration Dependency of Electronic Mobility}

Variation of low field electronic mobility as a function of acceptor concentration is shown in figure 2 for both $\mathrm{GaN}$ and $\mathrm{GaP}$, which also include the contribution of individual scattering mechanism in mobility.

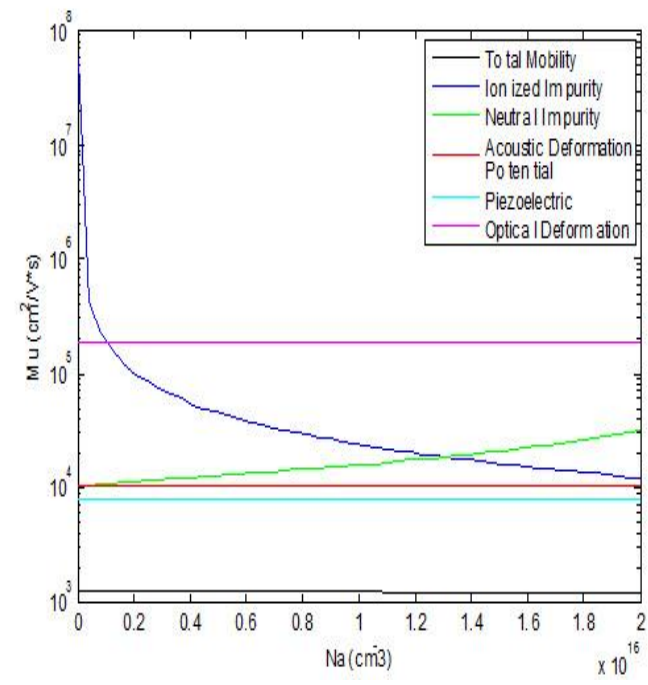

(a)

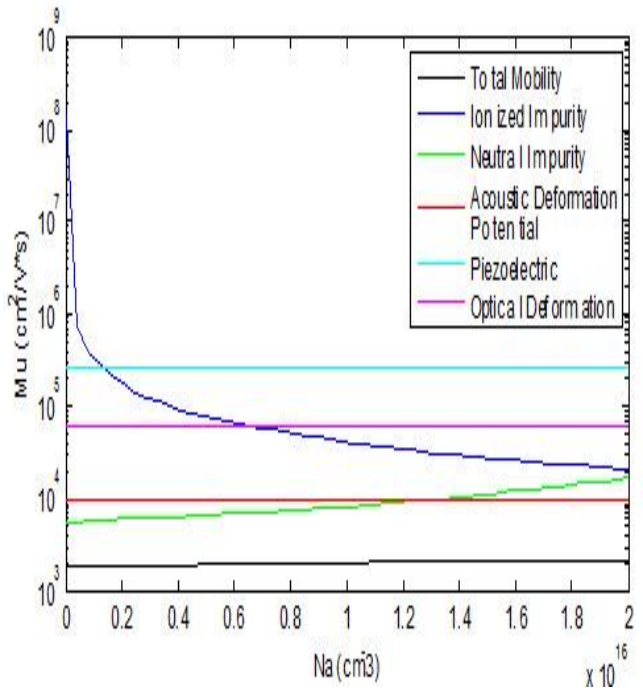

(b)

Figure 2. Acceptor concentration dependency of mobility, (a) for GaN and (b) for GaP.

\subsection{Donor Concentration Dependency of Electronic Mobility}

Variation of low field electronic mobility as a function of donor concentration is shown in figure 3 for both $\mathrm{GaN}$ and $\mathrm{GaP}$, which also include the contribution of individual scattering mechanism in mobility.

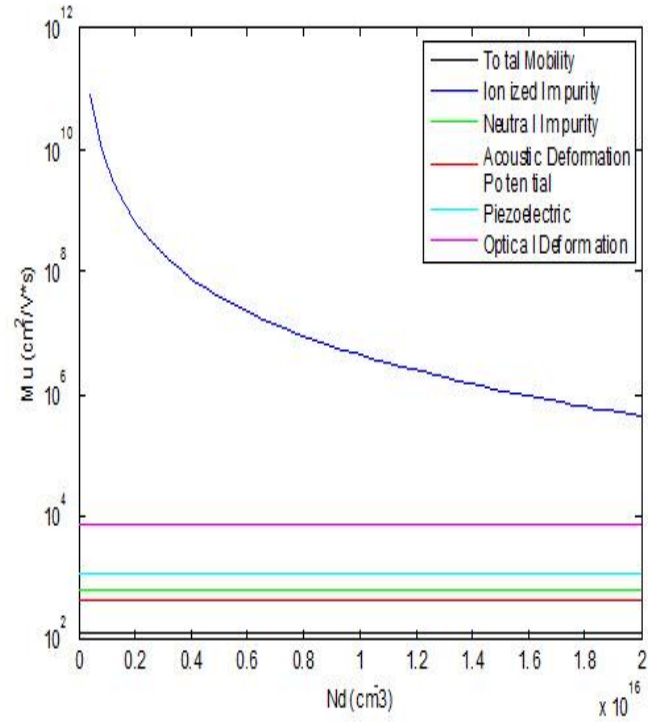

(a)

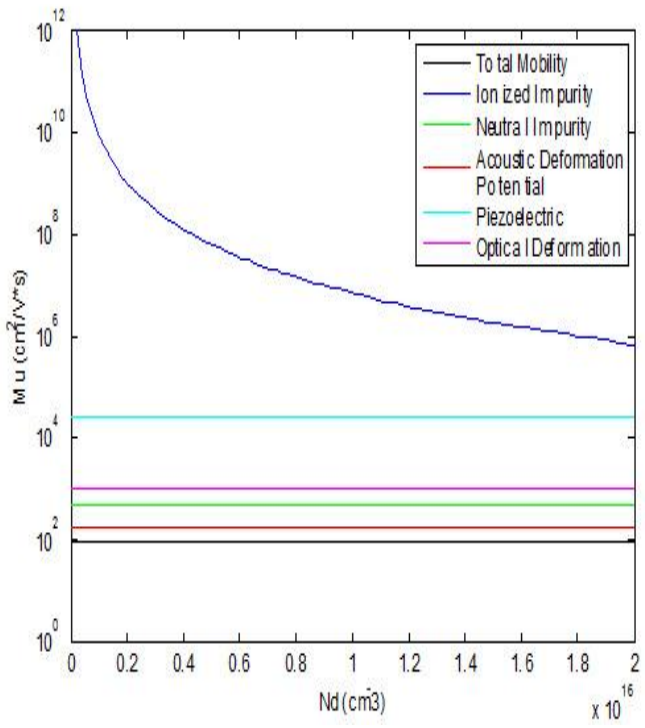

(b)

Figure 3. Donor concentration dependency of mobility, (a) for GaN and (b) for GaP. 


\section{CONCLuSion}

In summary, low field electron mobility has been calculated considering five different scattering mechanisms for GaN and InN. A number of scatterings have been omitted for simplicity. Calculation can be improved by considering all scatterings. Dependency of mobility upon temperature, donor concentration and acceptor concentration has been studied for both $\mathrm{GaN}$ and $\mathrm{GaP}$. The total electronic mobility decreases with increasing temperature for both $\mathrm{GaN}$ and $\mathrm{GaP}$. The total electronic mobility is almost independent of acceptor and donor concentration for both $\mathrm{GaN}$ and $\mathrm{GaP}$.

\section{REFERENCES}

[1] U. K. Mishra, L. Shen, T. E. Kazior and Y. F. Wu, "GaN-based RF power devices and amplifiers", invited paper, in Proc. IEEE 96 (2), 2008.

[2] U. K. Mishra and J. Singh, "Semiconductor device physics and design", The Netherlands: Springer, 2008.

[3] S. Nakamura, S. Pearton and G. Fasol, "The blue laser diode: The complete story", 2nd edition, Springer Veriag, NY, 2000.

[4] J. PipreK, "Nitride semiconductor devices: principles and devices", Germany: Wiley-VCH Verlag $\mathrm{GmbH} \& \mathrm{Co} . \mathrm{KGaA}, 2007$.

[5] J. R. S. D. C. Look, "Dislocation scattering in GaN", Physical Review Letters, 1999.

[6] D. Barrett, "A Program for calculating mobility and carrier density in bulk semiconductors",University of Notre Dame, USA.

[7] H. Morkoc, "Handbook of nitride semiconductors and devices: Electronic and optical processes in nitrides", Vol. 2, Germany: Wiley-VCH Verlag GmbH \& Co. KGaA, 2008.

[8] M. W. A. Wolfe, "Physical properties of semiconductors", Englewood Cliffs, N. J.: Prentice Hall, 1989.

[9] B. K. Ridley, "Quantum processes in semiconductor", 4th edition, Oxford Science Publications, Clarendon Press Oxford, 1999.

[10] K. Seeger, "Semiconductor physics", 9th edition, Berlin Heidelberg: Springer Verlag, 2004; Indian Reprint: 2007.

[11] B. L. Gelmonta M. Shur, and M. Stroscio, "Polar optical - phonon scattering in three - and two dimensional electron gases”, J. Appl. Phys., 1995. 